UCI-HEP-TR-2010-15

\title{
Constraints on Dark Matter from Colliders
}

\author{
Jessica Goodman, Masahiro Ibe, Arvind Rajaraman, \\ William Shepherd, Tim M.P. Tait and Hai-Bo Yu \\ Department of Physics and Astronomy, \\ University of California, Irvine, California 92697
}

(Dated: April 5, 2011)

\begin{abstract}
We show that colliders can impose strong constraints on models of dark matter, in particular when the dark matter is light. We analyze models where the dark matter is a fermion or scalar interacting with quarks and/or gluons through an effective theory containing higher dimensional operators which represent heavier states that have been integrated out of the effective field theory. We determine bounds from existing Tevatron searches for monojets as well as expected LHC reaches for a discovery. We find that colliders can provide information which is complementary or in some cases even superior to experiments searching for direct detection of dark matter through its scattering with nuclei. In particular, both the Tevatron and the LHC can outperform spin dependent searches by an order of magnitude or better over much of parameter space, and if the dark matter couples mainly to gluons, the LHC can place bounds superior to any spin independent search.
\end{abstract}




\section{INTRODUCTION}

While astrophysical observations provide compelling proof for the existence of a nonbaryonic dark component to the Universe and precise measurements as to its abundance [1], they offer no clue as to the mass of dark matter (DM) particles, how they fit into the Standard Model (SM) of particle physics, or even whether or not the dark matter has interactions beyond gravitational. The most compelling vision of dark matter is a weakly interacting massive particle (WIMP), which offers the possibility to understand the relic abundance of dark matter as a natural consequence of the thermal history of the Universe through the WIMP(less) miracle [2]. The large interactions of WIMPs with SM particles may imply detectable rates of WIMP annihilations into SM final states, scattering of WIMPs with heavy nuclei, and production of WIMPs in high energy reactions of SM particles at colliders.

WIMPs which produce signals in direct detection experiments must also couple to nucleons, and therefore can be produced at colliders like the Tevatron and LHC. Low mass particles are particularly amenable to searches at colliders, since the fact that a typical collision involves quarks and/or gluons carrying only a small fraction of the parent (anti-)proton energy implies that cross sections fall dramatically with the mass of produced states. Light states can thus be produced with very large rates. In the case of a WIMP, stability on the order of the lifetime of the Universe implies that pair production must highly dominate over single production, and precludes the WIMP from decaying within the detector volume. WIMPs therefore appear as missing energy, and can potentially be observed by searching for visible particles recoiling against dark matter particles [3 -7]. This can be used to set constraints on the WIMP couplings to the constituents of nuclei, which in turn can be translated to constraints on direct detection cross sections. In previous work [8], this was done for the case of Majorana WIMPs${ }^{1}$; here we extend this work to also include Dirac fermion and scalar (real or complex) WIMPs.

There is currently particular interest in light dark matter particles. The DAMA experiment has reported a signal of annual modulation at a high significance level [10]; this signal is consistent with a dark matter discovery interpretation with a dark matter parti-

\footnotetext{
${ }^{1}$ A partial set of operators for a Dirac WIMP were bounded using Tevatron data in Ref. [9] and the Tevatron limits and LHC reach for operator D8 (see below) were considered in Ref. [6] .
} 
cle of mass $\lesssim 10 \mathrm{GeV}[11,[12]$. The CoGeNT collaboration has also reported a signal [13] which can be explained by a WIMP in this mass range (though there may be some tension with unpublished data from 5 towers of CDMS Si detectors [14], and with recent data from XENON10/100 [15, 16]). There has hence been much recent interest in models of light dark matter (where the DM mass is order a few GeV) [17-26]. As colliders are most effective when producing highly boosted, light WIMPs, the tantalizing hints from DAMA and CoGeNT point toward a region where colliders can have a particular impact on theories of dark matter.

In this article, we consider the situation where the WIMP is the only new particle in the energy ranges relevant for current experiments. Given the small energy transfers involved in direct detection, this assumption is almost certainly justified. For colliders, the degree to which it is justified depends on the details of the WIMP theory. Under this assumption, the WIMP will couple to the SM particles through higher dimensional operators, presumably mediated by particles of the dark sector which are somewhat heavier than the WIMP itself (and which may or may not carry SM gauge charges).

This article is organized as follows. In Section II, we write down a complete list of leading operators and analyze the constraints on the coefficients of these operators, assuming that only one operator is dominant at a time. In Section III, we employ existing Tevatron and planned LHC searches to determine the constraints on the coefficients of the operators (or prospects for their discovery), respectively. Of the complete set of operators, some mediate substantial (i.e. not suppressed by the WIMP velocity) low energy WIMP-nucleus rates, and thus are constrained or may be discovered by direct detection experiments. These bounds and prospects are presented in Section IV. We conclude with comments on future directions in Section V.

\section{EFFECTIVE FIELD THEORY OF WIMP COUPLINGS}

We consider the cases where the DM particle is a scalar or a fermion; if a scalar, it can be real or complex, and if a fermion, it can be Majorana or Dirac. Each of these cases is considered separately. We note that in principle, the WIMP could also be spin one or higher; we shall not consider these cases here since the couplings of such WIMPs are usually restricted by gauge invariance and other symmetries, and are more heavily model-dependent. 
We shall be considering the situation where the WIMP (which we will generically denote $\chi)$ is the only particle in addition to the standard model fields accessible to colliders. We will assume that $\chi$ is odd under some $Z_{2}$ symmetry (e.g. R-parity in supersymmetry, or KKparity in extra dimensions), and hence each coupling involves an even number of WIMPs with the lowest dimensional operators we consider containing two WIMPs. We assume whatever particles mediate interactions between the WIMPs and the SM fields are somewhat heavier than the WIMPs themselves, with their leading effect manifest as higher dimensional operators in the effective field theory. For simplicity, we assume the WIMP is a singlet under the SM gauge groups, and thus possesses no tree-level couplings to the electroweak gauge bosons. We also neglect couplings with Higgs bosons. While the inclusion of such couplings in the effective theory is straightforward, we leave these cases for future work. Given the assumption that the WIMPs are SM singlets, the factor in each operator consisting of SM fields must also be invariant under SM gauge transformations.

We note in passing that even for an electroweak singlet WIMP, the lowest dimensional operator linking a pair of WIMPs to the SM fields contains two WIMPs and the SM Higgs bilinear $|H|^{2}[27]$. Such an interaction contributes to direct detection and collider processes involving WIMPs by inducing a $\chi-\chi-h^{0}$ interaction after electroweak symmetry-breaking ${ }^{2}$. While we do not consider this operator further, we note that for cases where the Higgs is heavy enough, it is effectively integrated out, leaving behind operators which we do consider.

The next allowed class of operators have SM factors which are quark or lepton bilinears. The lepton bilinear couplings contribute only at a very suppressed level to direct detection or hadron collider production, leaving us with little to say about them. It would be very interesting to study constraints on such operators from indirect detection experiments and/or lepton colliders such as LEP-II. In this note we shall focus on quark bilinear operators of the form $\bar{q} \Gamma q$, where $\Gamma$ is a $4 \times 4$ matrix of the complete set,

$$
\Gamma=\left\{1, \gamma^{5}, \gamma^{\mu}, \gamma^{\mu} \gamma^{5}, \sigma^{\mu \nu}\right\}
$$

We do not consider terms with derivatives acting on the quarks, which lead to higher dimensional operators, more suppressed at low energies.

Finally, we have operators mediating WIMP couplings to massless gauge fields. The leading operators are a magnetic moment coupling $\bar{\chi} \sigma^{\mu \nu} \chi F_{\mu \nu}$ and an electric dipole moment

\footnotetext{
${ }^{2}$ Collider and direct detection signals from this operator are explored in Ref. [28].
} 
coupling $\bar{\chi} \sigma^{\mu \nu} \gamma_{5} \chi F_{\mu \nu}$ (which are only non-vanishing for a Dirac fermion WIMP), though given the unbroken $U(1)_{E M}$ gauge invariance, they are likely induced at the loop level and thus may have small coefficients. Various experimental bounds and direct detection signals of these operators have been studied in Refs. [29 38]. We do not consider collider constraints for these operators further here and leave astrophysics bounds for future work [39]. We also have couplings to $G_{\mu \nu} G_{\alpha \beta}$, where $G G$ can either be a pair of electromagnetic or color field strengths, with gauge and Lorentz indices contracted in all possible ways to form a family of related operators. Here we focus on the operators involving color field strengths. Just as for quark operators, terms with derivatives acting on the gauge field strengths are higher order and more suppressed.

All together, these higher-dimensional operators define an effective field theory of the interactions of singlet WIMPs with hadronic matter. It is expected to reasonably capture the physics provided the WIMP is somewhat lighter than the particles which mediate its interactions with the SM. It is a non-renormalizable field theory and thus must break down at some energy scale, represented by the masses of those particles which have been integrated out. The quantities $M_{*}$ which characterize the interaction strength of the interactions are functions of the masses and the coupling strengths of the mediating particles to WIMPs and SM fields, and can be computed in terms of the fundamental parameters for any specific UV theory of interest.

What happens above the regime of validity of the effective theory depends on the UV completion, and is much more model dependent. Depending on the specifics of the UV theory, collider bounds may get stronger or weaker. For example, in supersymmetric theories our operators are UV completed into colored squarks which will be produced on-shell and may contribute to the jets + missing energy observable with larger rates than those we are computing in the effective theory. Other UV completions, such as a light neutral mediator, can lead to much weaker collider cross sections [9], since far above the mediator mass the rate will fall with jet transverse energy as $1 / P_{t}^{2}$, whereas in the effective theory the partonic reaction is flat with jet $P_{t}$, scaling as $1 / M_{*}^{2}$. Thus, it should be borne in mind that our limits strictly speaking only apply when all mediator masses are much larger than the typical energy of the reaction, and in the absence of a picture of the UV theory, it is hard to know whether the bounds are over- or under-estimated when the effective theory description does not strictly apply. 
For a given WIMP mass, there is a lower bound on $M_{*}$ such that one can imagine any weakly coupled UV completion. Since the operators mediate interactions with (at least) two colored SM fields coupled to two WIMPs, the simplest tree level UV completions have a single mediator particle and two interactions. The mapping to $M_{*}$ from the UV parameters thus involves an expression such as $M_{*} \sim M / \sqrt{g_{1} g_{2}}$ where $M$ is the mass of the exchanged particle, and $g_{1}$ and $g_{2}$ are couplings. Since an effective theory description requires $M>2 m_{\chi}$, and a preturbative theory $g_{1} g_{2} \lesssim(4 \pi)^{2}$, a weakly coupled UV completion requires $m_{\chi} \lesssim 2 \pi M_{*}$, beyond which the UV completion becomes non-perturbative. In determining bounds, since there is no imaginable perturbative UV picture for $m_{\chi} \lesssim 2 \pi M_{*}$, we cut off the bounded regions outside of this region of validity. Furthermore, for the effective theory to make sense, the mediator mass has to be larger than energy transfer through quarks at the collider environment. The limit, in which the effective theory breaks down, highly depends on the details of relevant patron energy and its distribution. Since $M \lesssim 4 \pi M_{*}$ for the perturbative UV completion, our bounds are valid when the characteristic energy transfer is smaller than $4 \pi M_{*}$. The detailed analysis of this limit is beyond the scope of this work, we will leave it for the future investigation.

The coefficients of the operators are chosen to simplify comparisons to direct detection experiments. For quark bilinears, the appropriate matrix elements (at low momentum transfer) are $\left\langle N\left|m_{q} \bar{q} q\right| N\right\rangle$ and $\left\langle N\left|\bar{q} \gamma^{\mu} q\right| N\right\rangle$ which contribute to spin-independent scattering, $\left\langle N\left|\bar{q} \gamma^{\mu} \gamma^{5} q\right| N\right\rangle$, which contributes to spin-dependent scattering, and $\left\langle N\left|\bar{q} \sigma^{\mu \nu} q\right| N\right\rangle$, which couples to the magnetic moment of the nucleon. For the gluon operators, the relevant matrix element is $\left\langle N\left|\alpha_{s} G G\right| N\right\rangle$. The scalar (and pseudo-scalar) quark bilinears are normalized by $m_{q}$, which together with our choice of universal vector-type couplings has the added feature of mitigating contributions to flavor changing processes from these operators, through the framework of minimal flavor violation [40]. For the gluon field strength operators, we normalize by a factor $\alpha_{s}$, which both anticipates their origin as loop processes and captures the dominant renormalization group evolution. The complete list of leading operators is given in Table I. The coefficients of these operators have been scaled by appropriate powers of $M_{*}$ (the value of which can be in principle different for each operator) to give the correct over-all dimension in the action. 


\begin{tabular}{|c|c|c|}
\hline Name & Operator & Coefficient \\
\hline D1 & $\bar{\chi} \chi \bar{q} q$ & $m_{q} / M_{*}^{3}$ \\
D2 & $\bar{\chi} \gamma^{5} \chi \bar{q} q$ & $i m_{q} / M_{*}^{3}$ \\
D3 & $\bar{\chi} \chi \bar{q} \gamma^{5} q$ & $i m_{q} / M_{*}^{3}$ \\
D4 & $\bar{\chi} \gamma^{5} \chi \bar{q} \gamma^{5} q$ & $m_{q} / M_{*}^{3}$ \\
D5 & $\bar{\chi} \gamma^{\mu} \chi \bar{q} \gamma_{\mu} q$ & $1 / M_{*}^{2}$ \\
D6 & $\bar{\chi} \gamma^{\mu} \gamma^{5} \chi \bar{q} \gamma_{\mu} q$ & $1 / M_{*}^{2}$ \\
D7 & $\bar{\chi} \gamma^{\mu} \chi \bar{q} \gamma_{\mu} \gamma^{5} q$ & $1 / M_{*}^{2}$ \\
D8 & $\bar{\chi} \gamma^{\mu} \gamma^{5} \chi \bar{q} \gamma_{\mu} \gamma^{5} q$ & $1 / M_{*}^{2}$ \\
D9 & $\bar{\chi} \sigma^{\mu \nu} \chi \bar{q} \sigma_{\mu \nu} q$ & $1 / M_{*}^{2}$ \\
D10 & $\bar{\chi} \sigma_{\mu \nu} \gamma^{5} \chi \bar{q} \sigma_{\alpha \beta} q$ & $i / M_{*}^{2}$ \\
D11 & $\bar{\chi} \chi G_{\mu \nu} G^{\mu \nu}$ & $\alpha_{s} / 4 M_{*}^{3}$ \\
D12 & $\bar{\chi} \gamma^{5} \chi G_{\mu \nu} G^{\mu \nu}$ & $i \alpha_{s} / 4 M_{*}^{3}$ \\
D13 & $\bar{\chi} \chi G_{\mu \nu} \tilde{G}^{\mu \nu}$ & $i \alpha_{s} / 4 M_{*}^{3}$ \\
D14 & $\bar{\chi} \gamma^{5} \chi G_{\mu \nu} \tilde{G}^{\mu \nu}$ & $\alpha_{s} / 4 M_{*}^{3}$ \\
\hline
\end{tabular}

\begin{tabular}{|c|c|c|}
\hline Name & Operator & Coefficient \\
\hline C1 & $\chi^{\dagger} \chi \bar{q} q$ & $m_{q} / M_{*}^{2}$ \\
C2 & $\chi^{\dagger} \chi \bar{q} \gamma^{5} q$ & $i m_{q} / M_{*}^{2}$ \\
C3 & $\chi^{\dagger} \partial_{\mu} \chi \bar{q} \gamma^{\mu} q$ & $1 / M_{*}^{2}$ \\
C4 & $\chi^{\dagger} \partial_{\mu} \chi \bar{q} \gamma^{\mu} \gamma^{5} q$ & $1 / M_{*}^{2}$ \\
C5 & $\chi^{\dagger} \chi G_{\mu \nu} G^{\mu \nu}$ & $\alpha_{s} / 4 M_{*}^{2}$ \\
C6 & $\chi^{\dagger} \chi G_{\mu \nu} \tilde{G}^{\mu \nu}$ & $i \alpha_{s} / 4 M_{*}^{2}$ \\
\hline R1 & $\chi^{2} \bar{q} q$ & $m_{q} / 2 M_{*}^{2}$ \\
R2 & $\chi^{2} \bar{q} \gamma^{5} q$ & $i m_{q} / 2 M_{*}^{2}$ \\
R3 & $\chi^{2} G_{\mu \nu} G^{\mu \nu}$ & $\alpha_{s} / 8 M_{*}^{2}$ \\
R4 & $\chi^{2} G_{\mu \nu} \tilde{G}^{\mu \nu}$ & $i \alpha_{s} / 8 M_{*}^{2}$ \\
\hline
\end{tabular}

TABLE I: Operators coupling WIMPs to SM particles. The operator names beginning with D, C, $\mathrm{R}$ apply to WIMPS that are Dirac fermions, complex scalars or real scalars respectively.

\section{COLLIDER CONSTRAINTS}

\section{A. Overview}

We can constrain $M_{*}$ for each operator in the table above by considering the pair production of WIMPs at a hadron collider:

$$
p \bar{p}(p p) \rightarrow \chi \chi+X
$$

Since the WIMPs escape undetected, this leads to events with missing transverse energy, recoiling against additional hadronic radiation present in the reaction.

The most significant Standard Model backgrounds to this process are events where a $Z$ boson decays into neutrinos, together with the associated production of jets. This background is irreducible. There are also backgrounds from events where a particle is either missed or has a mismeasured energy. The most important of these comes from events pro- 
ducing $W+$ jets, where the charged lepton from the $W$-decay is missed. Other backgrounds such as QCD multijet production (with the missing energy the result of mismeasuring the energy of one of more jets) are expected to be subdominant for the cuts chosen in the analyses [41, 42].

\section{B. Tevatron Constraints}

The Tevatron has searched for signals of new physics with missing transverse energy in many channels. We will focus on monojet events, where the WIMPs recoil against a single jet, with restrictions on any additional SM radiation. We will compare the predictions of our effective theories with the results on monojet events from CDF [42, 43]. We expect similar constraints can be derived from D0 data, but choose to focus on the available CDF searches which utilize much greater integrated luminosity. In Ref. [43], the events were required to satisfy:

- Events are required to have a leading Jet with transverse energy $E_{t}>80 \mathrm{GeV}$;

- Events must have net missing transverse energy $E_{t}>80 \mathrm{GeV}$;

- A second jet with $E_{t}<30 \mathrm{GeV}$ is allowed;

- Events containing additional jets with $E_{t}>20 \mathrm{GeV}$ are vetoed.

In order to simulate WIMP pair production events to compare to these bounds, we found the partonic cross section for $p \bar{p} \rightarrow j \chi \chi$ using Comphep [44, 45], where $j$ is any parton other than the top quark, and is required to have $E_{t}>80 \mathrm{GeV}$. At the parton level, this simultaneously requires that $E_{T}>80 \mathrm{GeV}$. We correct these parton-level estimates by an efficiency taking into account corrections from parton showering, hadronization, and energy smearing by the detector. This efficiency is computed by first hadronizing the generated partonlevel events using Pythia [46] (through the Comphep-Pythia interface [47]). The hadronized events are reconstructed at the detector level by passing them through PGS [48] tuned to simulate the response of the CDF detector ${ }^{3}$ and required to satisfy the detailed CDF analysis cuts. The efficiency is defined as the ratio of the number of events after the PGS-level

3 A previous study [6] found that this detector model was able to reproduce the backgrounds quoted in [43] to the few percent level. 
cuts to the number at the parton-level. We find that efficiencies range from $30 \%$ to $48 \%$ for WIMPs with various spins and masses ranging from $0-300 \mathrm{GeV}$. Given the relative insensitivity to the details, we choose a flat efficiency of $40 \%$ for all cases.

We plot $2 \sigma$ lower limits on the scale of new physics $M_{*}$ for each operator as solid lines in figures 8,16, where for illustration, we also plot the lines resulting in the observed thermal relic density. Comparing with previous studies which analyzed D8 [6] and D1, D4, D5, and D8 (but with a different normalization between different flavors of quarks) [9], we find rough agreement with these studies. It is worth noting that the CDF analysis was somewhat optimized for theories with large extra dimensions as opposed to $\chi \chi j$, and it is possible that better bounds may be available for more optimized analysis strategies.

\section{LHC Prospects}

We also simulate the inclusive jets and missing transverse energy events at the LHC experiments for $\sqrt{s}=14 \mathrm{TeV}$ and compare them with the analysis in Ref. [49], which studied this signal in the context of a large extra dimensions search. In Ref. [49], the missing $E_{t}$ was required to be larger than $500 \mathrm{GeV}$ and no veto on additional hadronic radiation was imposed. Additional hard isolated leptons were vetoed in order to reduce backgrounds from $W+$ jets processes. Finally, the azimuthal angle between the the missing transverse energy and the second hardest jet was required to be $\Delta \Phi \geq 0.5$, to mitigate the QCD background due to mismeasured jet energies. The expected number of background events after these cuts was simulated to be about $B=2 \times 10^{4}$ with $100 \mathrm{fb}^{-1}$ of data.

Proceeding as before, we find the parton-level cross section $\sigma_{j \chi \chi}$ (with $E_{t}$ of the jet greater than $500 \mathrm{GeV}$ ) using Comphep. We use Pythia and the PGS simulation with the generic LHC detector model to estimate an efficiency, defined as for the Tevatron study, of roughly $80 \%$. This is roughly in agreement with the efficiency for the monojet signal from the large extra dimension model, which was found in Ref. [49] to be $\sim 90 \%$.

We define the $5 \sigma$ detection region for which the number of expected signal events $S$ and background events $B$ satisfy $S / \sqrt{B} \geq 5$ for an integrated luminosity of $100 \mathrm{fb}^{-1}$. We plot this $5 \sigma$ reach as dashed lines in figures 8, 16. Again, we note that this search was optimized for a large extra dimensions signal, and our knowledge of the LHC detector performance and SM

backgrounds are expected to substantially improve over those available to Ref. [49]. Given 
our positive results, a dedicated reanalysis by the collaborations would be very interesting.

\section{IMPLICATIONS FOR DIRECT DETECTION}

Our new bounds on the strength of interactions of WIMPs with hadrons can be translated into constraints on the possible contributions to direct detection cross sections for each of those interactions. Only some operators contribute to direct detection in the limit of low momentum transfer, and the remaining operators are suppressed by powers of the WIMP velocity, generically expected to be of order $\sim 10^{-3}$. For each contributing operator we employ the expectation value of the partonic operator in the nucleon [50]. This, combined with the kinematics of WIMP-nucleon scattering, results in cross sections

$$
\begin{aligned}
\sigma_{0}^{D 1} & =1.60 \times 10^{-37} \mathrm{~cm}^{2}\left(\frac{\mu_{\chi}}{1 \mathrm{GeV}}\right)^{2}\left(\frac{20 \mathrm{GeV}}{M_{*}}\right)^{6}, \\
\sigma_{0}^{D 5, C 3} & =1.38 \times 10^{-37} \mathrm{~cm}^{2}\left(\frac{\mu_{\chi}}{1 \mathrm{GeV}}\right)^{2}\left(\frac{300 \mathrm{GeV}}{M_{*}}\right)^{4}, \\
\sigma_{0}^{D 8, D 9} & =9.18 \times 10^{-40} \mathrm{~cm}^{2}\left(\frac{\mu_{\chi}}{1 \mathrm{GeV}}\right)^{2}\left(\frac{300 \mathrm{GeV}}{M_{*}}\right)^{4}, \\
\sigma_{0}^{D 11} & =3.83 \times 10^{-41} \mathrm{~cm}^{2}\left(\frac{\mu_{\chi}}{1 \mathrm{GeV}}\right)^{2}\left(\frac{100 \mathrm{GeV}}{M_{*}}\right)^{6}, \\
\sigma_{0}^{C 1, R 1} & =2.56 \times 10^{-36} \mathrm{~cm}^{2}\left(\frac{\mu_{\chi}}{1 \mathrm{GeV}}\right)^{2}\left(\frac{10 \mathrm{GeV}}{m_{\chi}}\right)^{2}\left(\frac{10 \mathrm{GeV}}{M_{*}}\right)^{4}, \\
\sigma_{0}^{C 5, R 3} & =7.40 \times 10^{-39} \mathrm{~cm}^{2}\left(\frac{\mu_{\chi}}{1 \mathrm{GeV}}\right)^{2}\left(\frac{10 \mathrm{GeV}}{m_{\chi}}\right)^{2}\left(\frac{60 \mathrm{GeV}}{M_{*}}\right)^{4} .
\end{aligned}
$$

where $\mu_{\chi}$ is the reduced mass of the WIMP-nucleon system.

The behavior at low WIMP masses is affected strongly by the spin of the WIMP itself. For a fermion WIMP the direct detection cross section for a fixed coupling is largely flat as the WIMP mass is decreased, until the WIMP is lighter than the nucleon mass. For a scalar WIMP, except for the vector-type interaction C3, the mass appears explicitly in the expression for the cross section, causing the cross section for smaller WIMP masses to notably increase (provided $m_{\chi}<M_{*}$ ). This has the net effect of weakening the impact of the collider bounds on the direct detection parameter space for of very light scalar dark matter with respect to those for fermion dark matter.

We also notice that the collider bounds on direct detection of non-self-conjugate fields are stronger by a factor of 2 in cross section than those on self-conjugate fields. This is an 
expected result, as the phase space for direct detection is unchanged by this factor but the phase space of collider production is suppressed by a factor of 2 for self-conjugate fields.

We plot the effective collider constraints on the WIMP direct detection parameter space in figures 177, including the most relevant direct detection constraints for comparison. We see that in all cases, colliders can probe regions of very light WIMP masses more effectively than direct detection experiments, which become limited by energy thresholds for extremely light WIMPs. Indeed, for many operators, the direct detection rates are expected to be very small because of the velocity suppression, and colliders become the only way to effectively probe WIMP-hadron interactions. In the case of a WIMP whose dominant recoil is through a spin-dependent interaction, collider constraints are already much stronger even than the expected reaches of near-future direct detection experiments. Thus, if such an experiment were to observe a positive signal, the collider constraints would immediately imply a breakdown of the effective field theory at collider energies, revealing the existence of a light mediator particle.

\section{CONCLUSIONS AND OUTLOOK}

We have studied constraints on dark matter models coming from collider experiments, specifically from extant searches at the Tevatron and future searches at the LHC. This extends our previous work where we considered the case of dark matter particles which were Majorana fermions to the cases where the dark matter is a Dirac fermion or a scalar (either real or complex).

Our results are qualitatively similar to our previous paper. In general collider constraints are very strong for lighter dark matter and fall off when the dark matter mass exceeds the typical energy reach of the collider. The constraints also depend on the coupling of the dark

matter; if the dark matter primarily couples to gluons, the constraints from colliders become especially strong.

One of the most interesting results is that collider constraints on spin dependent interactions are stronger than direct searches over a significant portion of parameter space. In the event that direct searches find a signal in this region while no signal is found at colliders, this will suggest that dark matter is a WIMP of spin 1 or higher, or that there exists a light mediator particle UV completing the interaction operators in such a way as to weaken the 


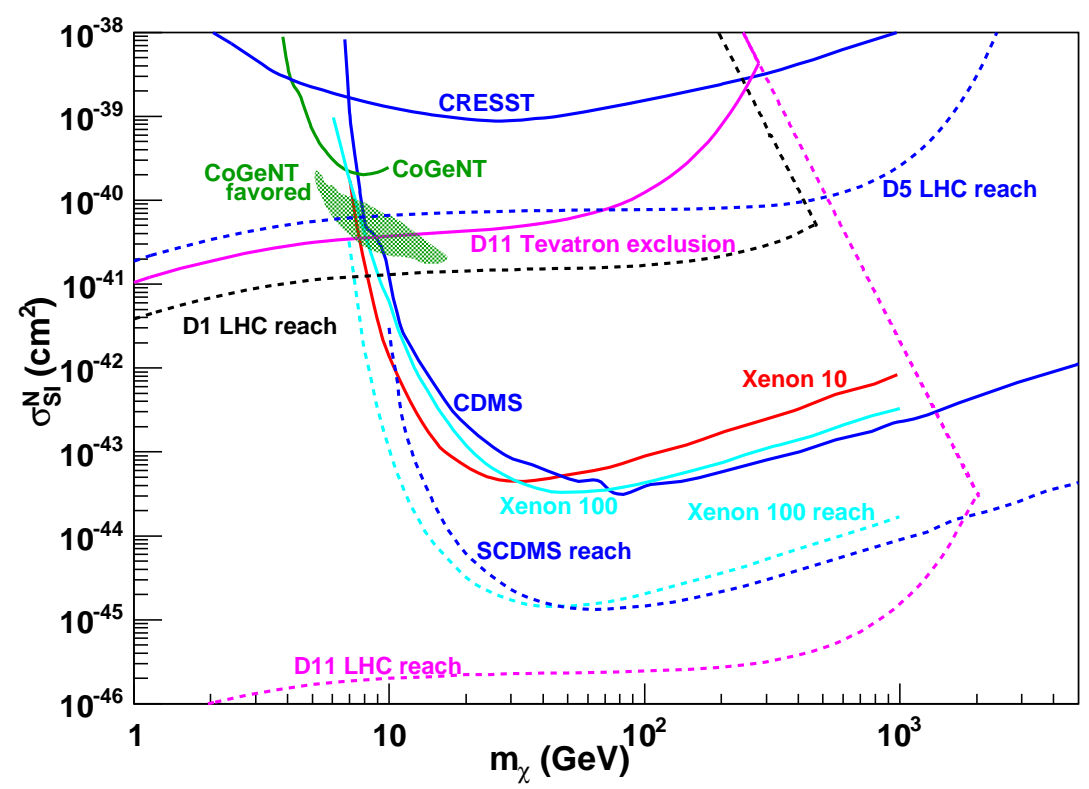

FIG. 1: Current experimental limits on spin-independent WIMP direct detection from CRESST 52], CDMS [53], Xenon 10 [54], CoGeNT [13], and Xenon 100 [15], (solid lines as labeled), as well as the CoGeNT favored region [13] and future reach estimates for SCDMS [55] and Xenon 100 [56], where we have chosen the line using a threshold of $3 \mathrm{PE}$ and the conservative extrapolation of $\mathcal{L}_{\text {eff }}$ (dashed lines as labeled). Also shown are the current Tevatron exclusion for the operator D11 (solid magenta line) as well as LHC discovery reaches (dashed lines as labeled) for relevant operators.

collider bounds. The case of a light mediator with a particular

$$
\text { dark matter }+ \text { dark matter } \leftrightarrow \text { SM-neutral mediator } \leftrightarrow \text { SM }+ \text { SM }
$$

completion structure was considered in [9]. Beyond these particular constructions, many models have additional light states which UV complete the interactions between the dark matter and the Standard Model through a

$$
\text { dark matter }+\mathrm{SM} \leftrightarrow \mathrm{SM} \text {-charged mediator } \leftrightarrow \text { dark matter }+\mathrm{SM}
$$

topology. It would be relatively simple to consider a complete set (as dictated by SM gauge and Lorentz invariance) of UV completions, and it would be interesting to see how our bounds are modified in the presence of such new states, and whether new collider signals 


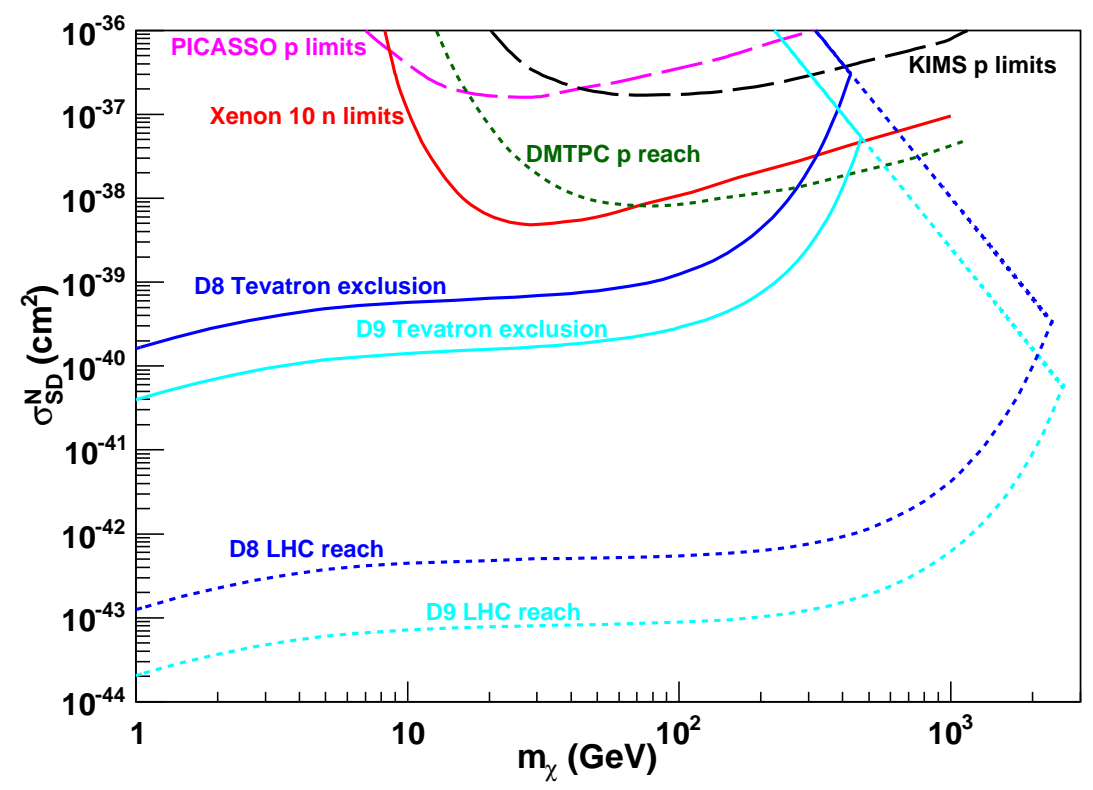

FIG. 2: Current experimental limits on spin-dependent WIMP direct detection from Picasso [57], KIMS [58], and Xenon 10 [54], as well as the future reach of DMTPC [59]. Also shown are the current Tevatron exclusions (solid lines as labeled) and LHC discovery reaches (dashed lines as labeled) for relevant operators.

can be found to constrain such models. We leave detailed exploration of these issues for future work.

Finally, we note that while effective theories may not always capture our favorite parameters of our favorite UV-complete models, they do provide a language to describe WIMP-SM interactions which captures a wide class of theories in a fairly model-independent fashion.

\section{Acknowledgements}

T. Tait is glad to acknowledge conversations with M. Beltran, P. Fox, D. Hooper, E. W. Kolb, Z. Krusberg, J. Wacker. and the hospitality of the SLAC theory group, for their generosity during his many visits. The authors are grateful to E.W. Kolb and Z. Kruseberg for catching an error in Eq. (7) in an earlier version of the draft. The work of AR and MI is supported in part by NSF grant PHY-0653656. The work of HY is supported in part by 


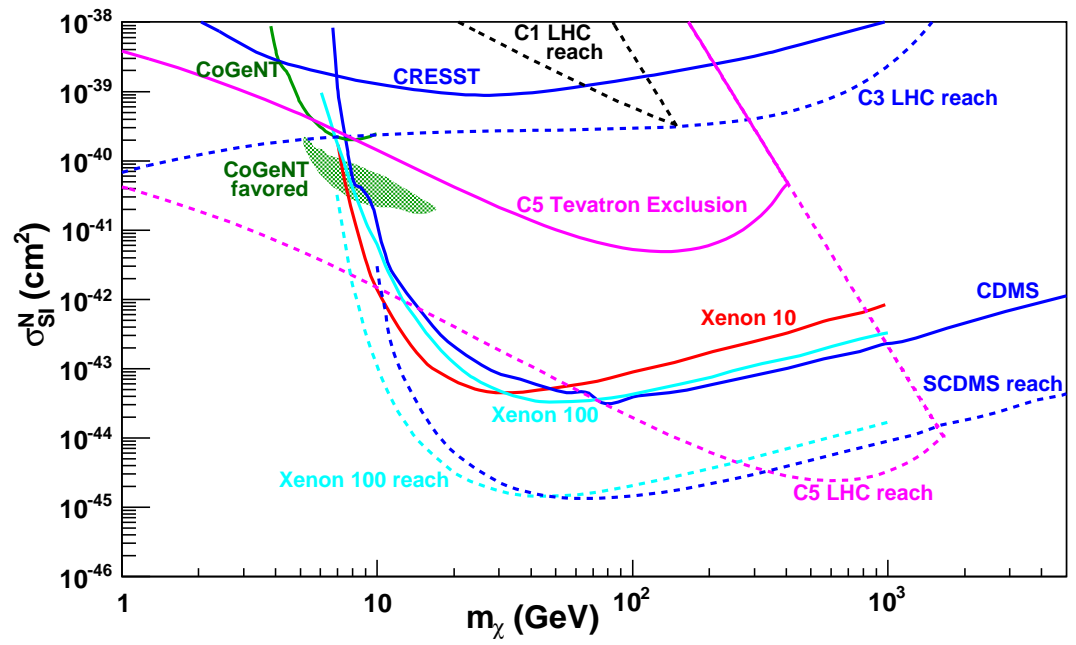

FIG. 3: Same as Fig.1, but with Tevatron exclusions and LHC reaches for complex scalar WIMP operators, as labeled.

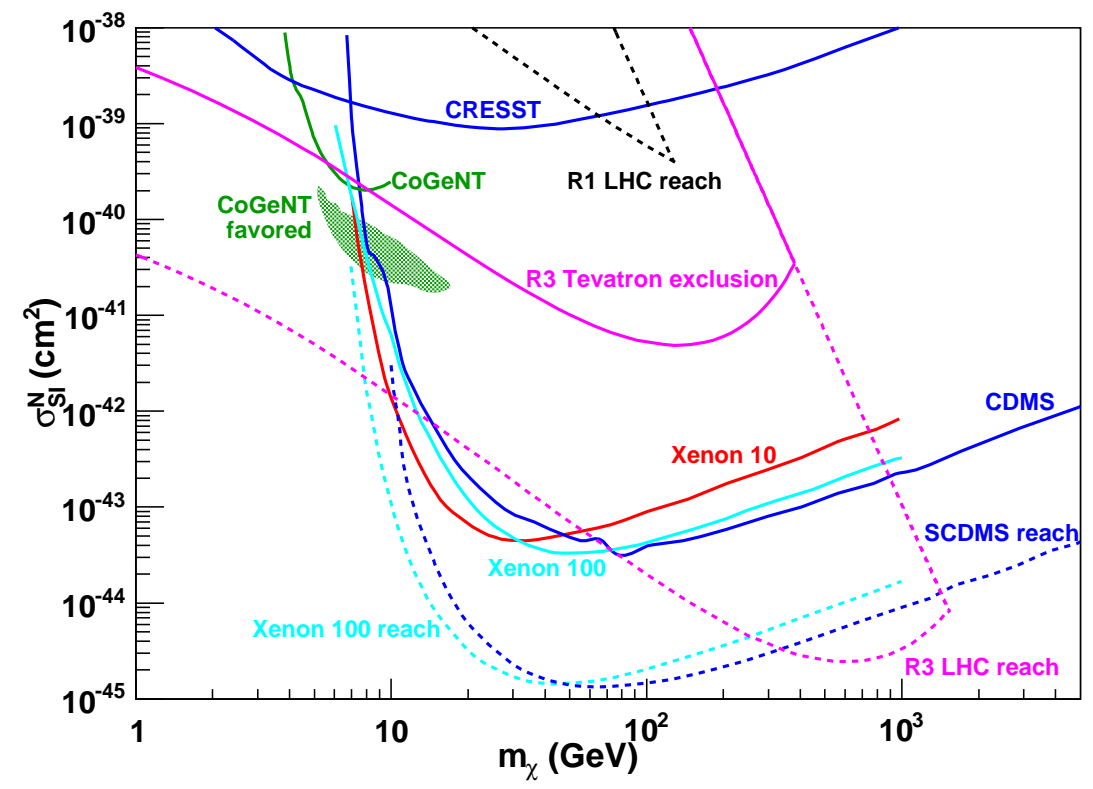

FIG. 4: Same as Fig.1, but with Tevatron exclusions and LHC reaches for real scalar WIMP operators, as labeled. 


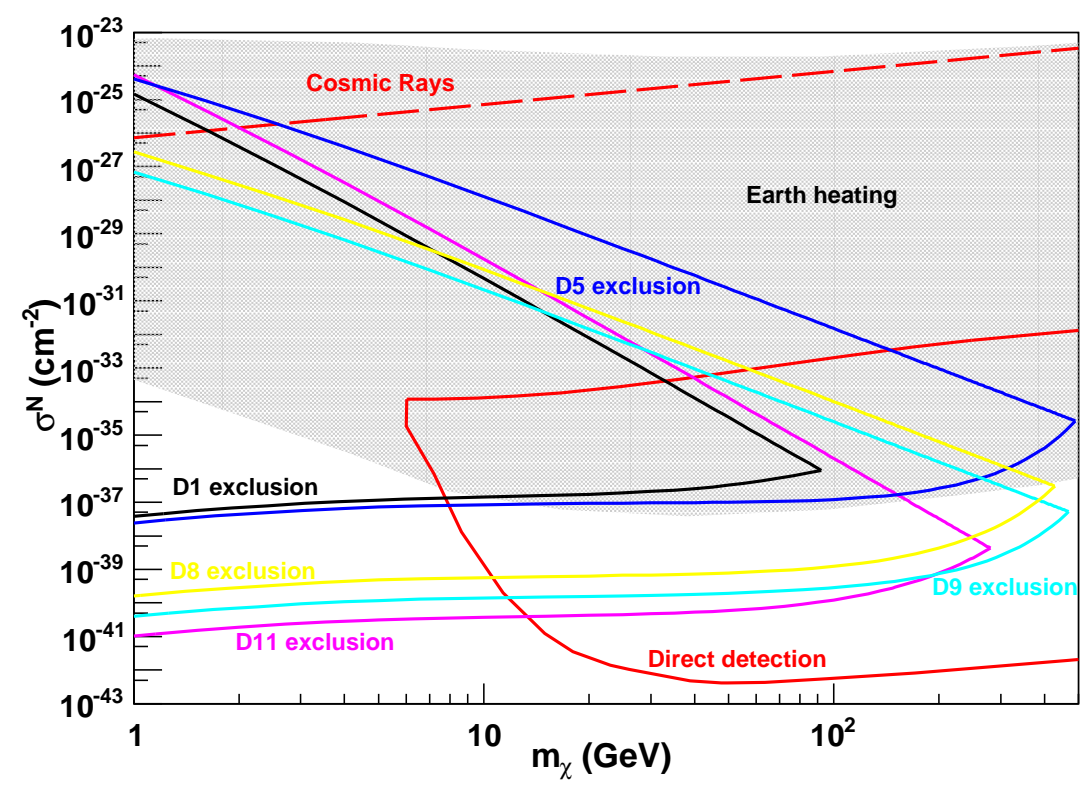

FIG. 5: A cartoon representation of previous limits due to direct detection experiments as well as constraints from Earth heating and cosmic rays [51], with new exclusions from Tevatron searches for Dirac WIMPs superimposed.

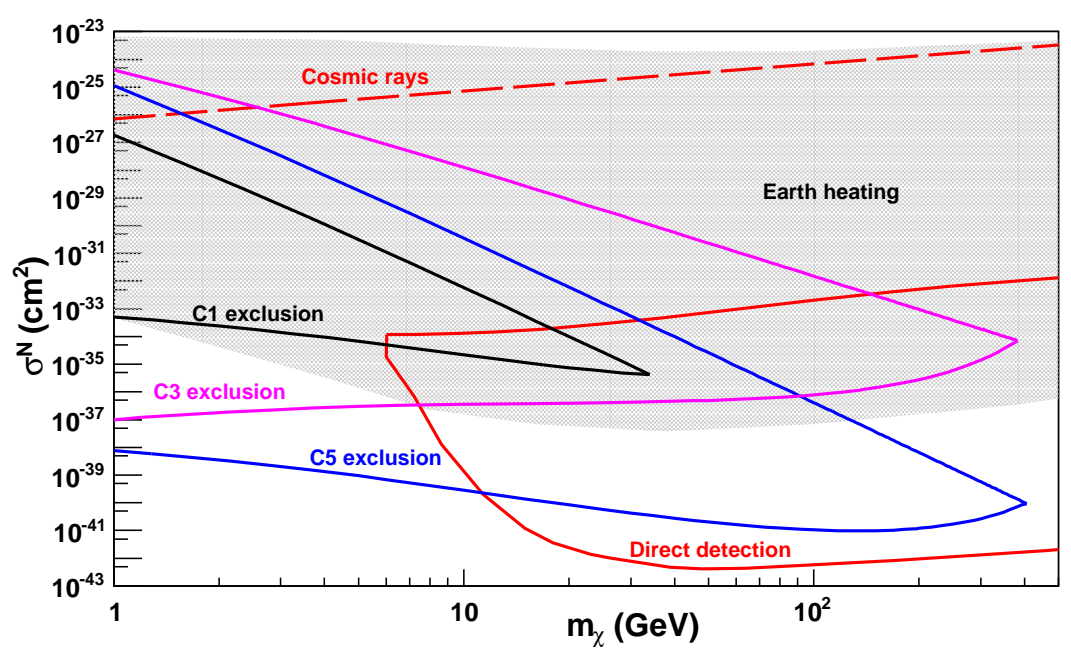

FIG. 6: Same as Fig.5, but with complex scalar WIMP limits superimposed. 


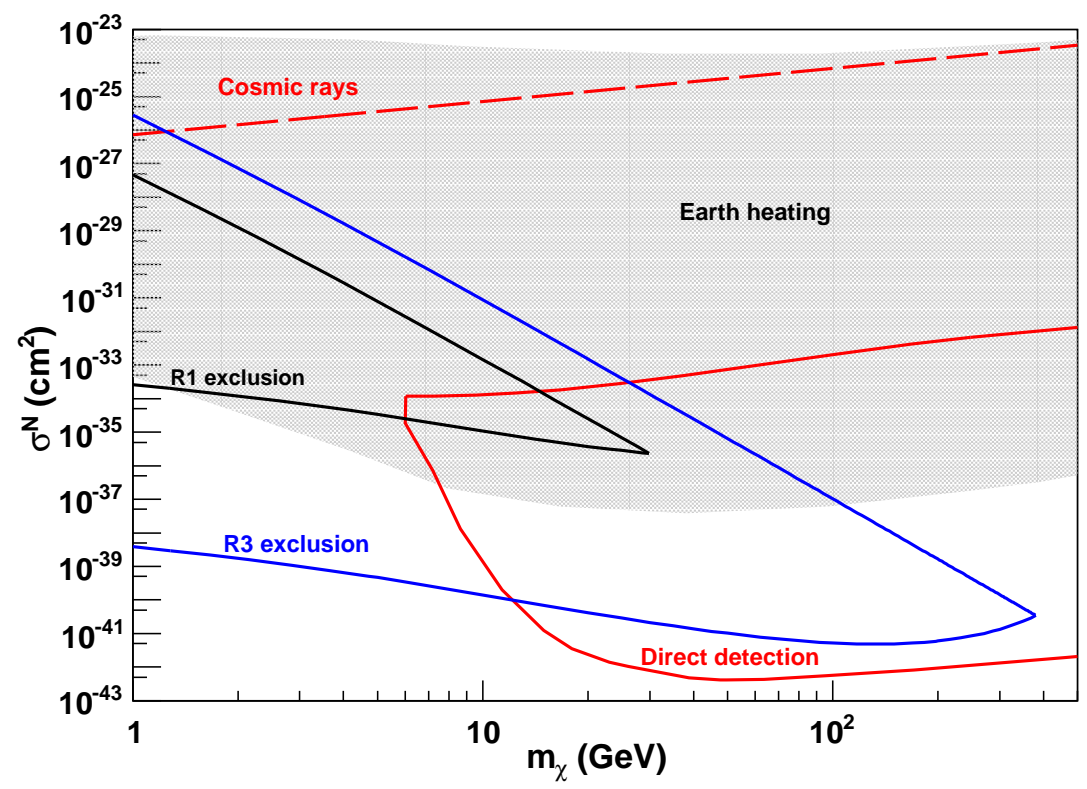

FIG. 7: Same as Fig. 5 , but with real scalar WIMP limits superimposed.

NSF grants PHY-0653656 and PHY-0709742.

[1] E. Komatsu et al., arXiv:1001.4538 [astro-ph.CO].

[2] J. L. Feng and J. Kumar, Phys. Rev. Lett. 101, 231301 (2008) arXiv:0803.4196 [hep-ph]]. J. L. Feng, H. Tu and H. B. Yu, JCAP 0810 (2008) 043 [arXiv:0808.2318 [hep-ph]].

[3] A. Birkedal, K. Matchev and M. Perelstein, Phys. Rev. D 70, 077701 (2004) arXiv:hep-ph/0403004]; P. Konar, K. Kong, K. T. Matchev and M. Perelstein, New J. Phys. 11, 105004 (2009) arXiv:0902.2000 [hep-ph]]; J. L. Feng, S. Su and F. Takayama, Phys. Rev. Lett. 96, 151802 (2006) arXiv:hep-ph/0503117.

[4] M. Beltran, D. Hooper, E. W. Kolb and Z. C. Krusberg, Phys. Rev. D 80, 043509 (2009) arXiv:0808.3384 [hep-ph]].

[5] Q. H. Cao, C. R. Chen, C. S. Li and H. Zhang, arXiv:0912.4511 [hep-ph].

[6] M. Beltran, D. Hooper, E. W. Kolb, Z. A. C. Krusberg and T. M. P. Tait, arXiv:1002.4137 [hep-ph].

[7] W. Shepherd, T. M. P. Tait and G. Zaharijas, Phys. Rev. D 79, 055022 (2009) arXiv:0901.2125 [hep-ph]]. 
[8] J. Goodman, M. Ibe, A. Rajaraman, W. Shepherd, T. M. P. Tait and H. B. P. Yu, arXiv:1005.1286 [hep-ph];

[9] Y. Bai, P. J. Fox and R. Harnik, arXiv:1005.3797 [hep-ph].

[10] R. Bernabei et al., arXiv:1002.1028 [astro-ph.GA].

[11] F. Petriello and K. M. Zurek, JHEP 0809, 047 (2008) arXiv:0806.3989 [hep-ph]].

[12] J. L. Feng, J. Kumar and L. E. Strigari, Phys. Lett. B 670, 37 (2008) arXiv:0806.3746 [hep-ph]].

[13] C. E. Aalseth et al. [CoGeNT collaboration], arXiv:1002.4703 [astro-ph.CO].

[14] J. Filippini, "WIMP Hunting with the Cryogenic Dark Matter Search", Les Rencontres de Physique de la Val le dAosta (2009).

[15] E. Aprile et al. [XENON100 Collaboration], arXiv:1005.0380 [astro-ph.CO].

[16] P. Sorensen, arXiv:1007.3549 [astro-ph.IM].

[17] Y. G. Kim and S. Shin, JHEP 0905, 036 (2009) arXiv:0901.2609 [hep-ph]].

[18] A. L. Fitzpatrick, D. Hooper and K. M. Zurek, arXiv:1003.0014 [hep-ph];

D. Feldman, Z. Liu and P. Nath, arXiv:1003.0437 [hep-ph].

[19] J. Kopp, T. Schwetz and J. Zupan, JCAP 1002, 014 (2010) arXiv:0912.4264 [hep-ph]].

[20] E. Kuflik, A. Pierce and K. M. Zurek, arXiv:1003.0682 [hep-ph].

[21] S. Andreas, C. Arina, T. Hambye, F. S. Ling and M. H. G. Tytgat, arXiv:1003.2595 [hep-ph]; K. J. Bae, H. D. Kim and S. Shin, arXiv:1005.5131 [hep-ph].

[22] S. Chang, J. Liu, A. Pierce, N. Weiner and I. Yavin, arXiv:1004.0697 [hep-ph].

[23] R. Essig, J. Kaplan, P. Schuster and N. Toro, arXiv:1004.0691 [hep-ph];

P. W. Graham, R. Harnik, S. Rajendran and P. Saraswat, arXiv:1004.0937 [hep-ph].

[24] H. An, S. L. Chen, R. N. Mohapatra, S. Nussinov and Y. Zhang, Phys. Rev. D 82, 023533 (2010) arXiv:1004.3296 [hep-ph]].

[25] V. Barger, M. McCaskey and G. Shaughnessy, arXiv:1005.3328 [hep-ph].

[26] D. Hooper, J. I. Collar, J. Hall and D. McKinsey, arXiv:1007.1005 [hep-ph].

[27] C. P. Burgess, M. Pospelov and T. ter Veldhuis, Nucl. Phys. B 619, 709 (2001) arXiv:hep-ph/0011335; H. Davoudiasl, R. Kitano, T. Li and H. Murayama, Phys. Lett. B 609, 117 (2005) (arXiv:hep-ph/0405097).

[28] S. Kanemura, S. Matsumoto, T. Nabeshima and N. Okada, arXiv:1005.5651 [hep-ph].

[29] K. Sigurdson, M. Doran, A. Kurylov, R. R. Caldwell and M. Kamionkowski, Phys. Rev. D 
70, 083501 (2004) [Erratum-ibid. D 73, 089903 (2006)] arXiv:astro-ph/0406355].

[30] J. Bagnasco, M. Dine and S. D. Thomas, Phys. Lett. B 320, 99 (1994) arXiv:hep-ph/9310290.

[31] M. Pospelov and T. ter Veldhuis, Phys. Lett. B 480, 181 (2000) arXiv:hep-ph/0003010.

[32] S. Gardner, Phys. Rev. D 79, 055007 (2009) arXiv:0811.0967 [hep-ph]].

[33] E. Masso, S. Mohanty and S. Rao, Phys. Rev. D 80, 036009 (2009) [arXiv:0906.1979 [hep-ph]].

[34] W. S. Cho, J. H. Huh, I. W. Kim, J. E. Kim and B. Kyae, Phys. Lett. B 687, 6 (2010) arXiv:1001.0579 [hep-ph]].

[35] S. Chang, N. Weiner and I. Yavin, arXiv:1007.4200 [hep-ph].

[36] V. Barger, W. Y. Keung and D. Marfatia, arXiv:1007.4345 [hep-ph].

[37] T. Banks, J. F. Fortin and S. Thomas, arXiv:1007.5515 [hep-ph].

[38] A. L. Fitzpatrick and K. M. Zurek, arXiv:1007.5325 [hep-ph].

[39] J. Goodman, M. Ibe, A. Rajaraman, W. Shepherd, T.M.P. Tait and H.-B. Yu, in preparation.

[40] A. J. Buras, P. Gambino, M. Gorbahn, S. Jager and L. Silvestrini, Phys. Lett. B 500, 161 (2001) arXiv:hep-ph/0007085.

[41] J. Alwall, M. P. Le, M. Lisanti and J. G. Wacker, Phys. Rev. D 79, 015005 (2009) arXiv:0809.3264 [hep-ph]].

E. Izaguirre, M. Manhart and J. G. Wacker, arXiv:1003.3886 [hep-ph].

[42] T. Aaltonen et al. [CDF Collaboration], Phys. Rev. Lett. 101, 181602 (2008) arXiv:0807.3132 [hep-ex]].

[43] http://www-cdf.fnal.gov/physics/exotic/r2a/20070322.monojet/public/ykk.html

[44] A. Pukhov et al., arXiv:hep-ph/9908288.

[45] E. Boos et al. [CompHEP Collaboration], Nucl. Instrum. Meth. A 534, 250 (2004) arXiv:hep-ph/0403113.

[46] T. Sjostrand, S. Mrenna and P. Z. Skands, JHEP 0605, 026 (2006) arXiv:hep-ph/0603175].

[47] A. S. Belyaev et al., arXiv:hep-ph/0101232,

[48] http://www.physics.ucdavis.edu/ conway/research/software/pgs/pgs4-general.htm

[49] L. Vacavant and I. Hinchliffe, J. Phys. G 27, 1839 (2001).

[50] G. Bèlanger, F. Boudjema, A. Pukhov, and A. Semenov, Comput.Phys.Commun.180:747767,2009 [arXiv:0803.2360v2 [hep-ph]]

[51] G. D. Mack, J. F. Beacom and G. Bertone, Phys. Rev. D 76, 043523 (2007) arXiv:0705.4298 [astro-ph]]. 
[52] G. Angloher et al., Astropart. Phys. 18, 43 (2002).

[53] Z. Ahmed et al. [The CDMS-II Collaboration], arXiv:0912.3592 [astro-ph.CO].

[54] J. Angle et al. [XENON Collaboration], Phys. Rev. Lett. 100, 021303 (2008) arXiv:0706.0039 [astro-ph]].

[55] D. S. Akerib et al., Nucl. Instrum. Meth. A 559, 411 (2006).

[56] E. Aprile, L. Baudis and f. t. X. Collaboration, PoS IDM2008, 018 (2008) arXiv:0902.4253 [astro-ph.IM]].

[57] S. Archambault et al., Phys. Lett. B 682, 185 (2009) arXiv:0907.0307 [hep-ex]].

[58] H. S. Lee et al. [KIMS Collaboration], Phys. Rev. Lett. 99, 091301 (2007) arXiv:0704.0423 [astro-ph]].

[59] G. Sciolla et al., J. Phys. Conf. Ser. 179, 012009 (2009) arXiv:0903.3895 [astro-ph.IM]].

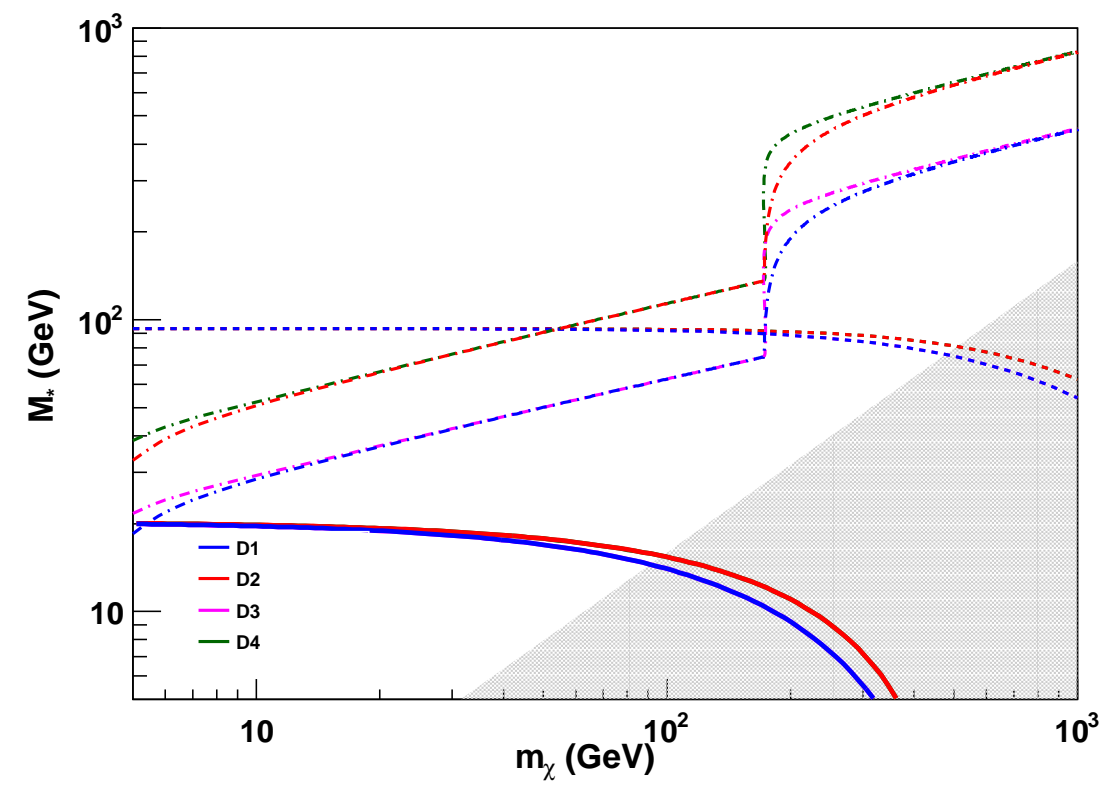

FIG. 8: Constraints on the operators D1-4. Solid lines are Tevatron $2 \sigma$ lower limits, dashed lines are LHC $5 \sigma$ discovery reach lower limits, and dot-dashed lines indicate the values of $M_{*}$ necessary for the WIMP to have the correct relic abundance in absence of any other interactions. The curves for operators D1 and D2 are largely degenerate with those for D3 and D4, respectively. The gray filled region indicates where the effective field theory breaks down, possessing no simple perturbative UV completion. 


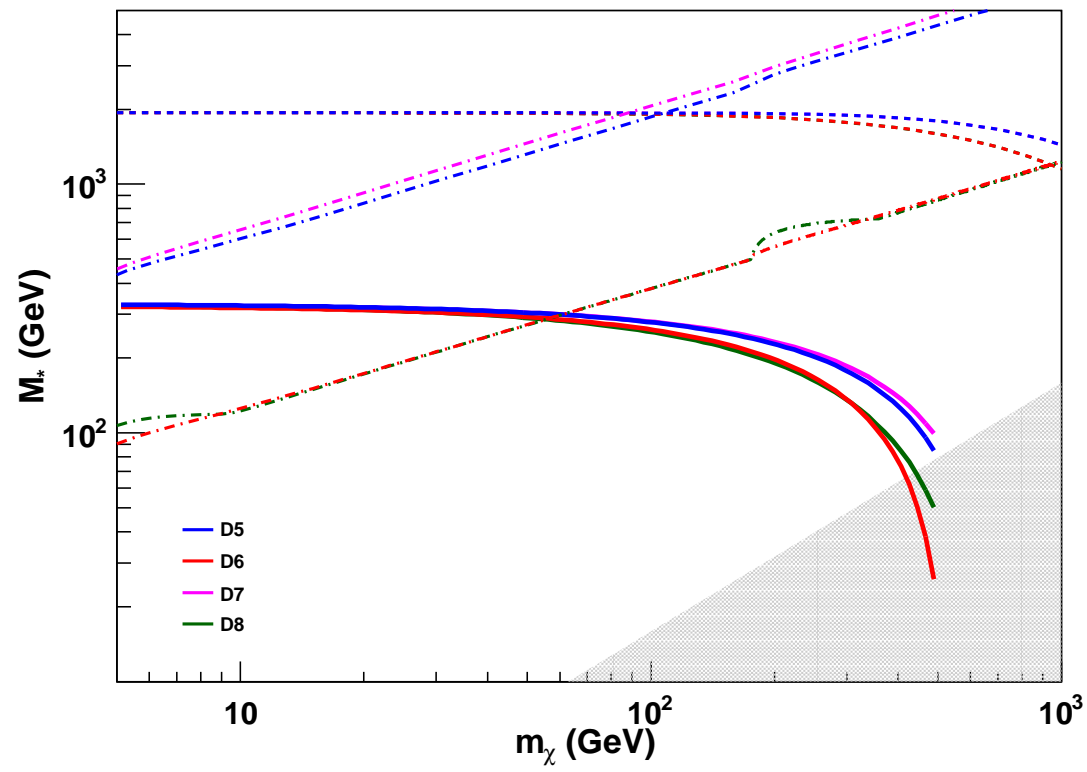

FIG. 9: Same as Fig.8, but for the operators D5 and D6 which are largely degenerate with D7 and D8, respectively.

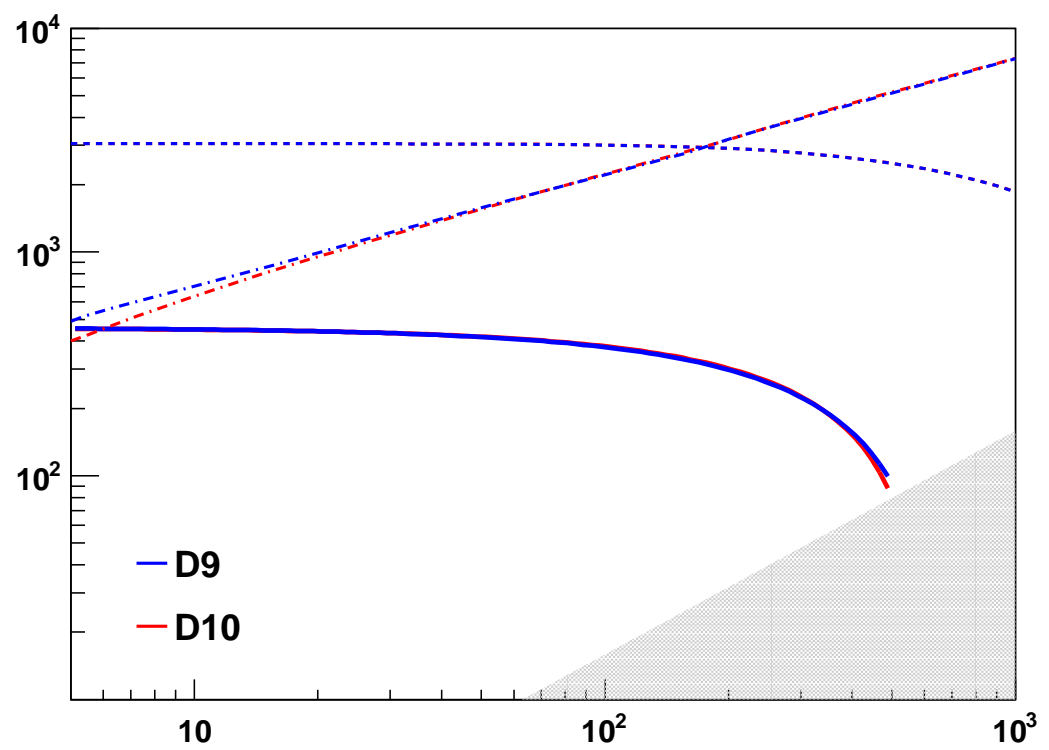

FIG. 10: Same as Fig.8, but for the operators D9 and D10. 


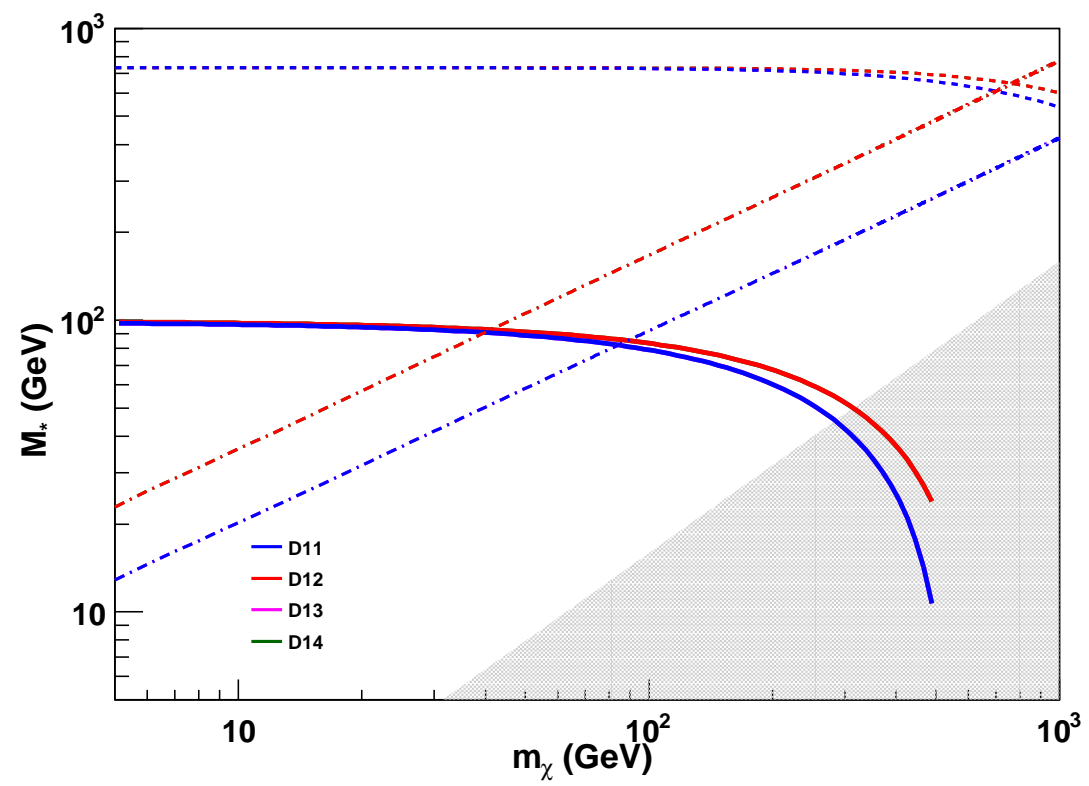

FIG. 11: Same as Fig.8, but for the operators D11 and D12 which are largely degenerate with D13 and D14, respectively.

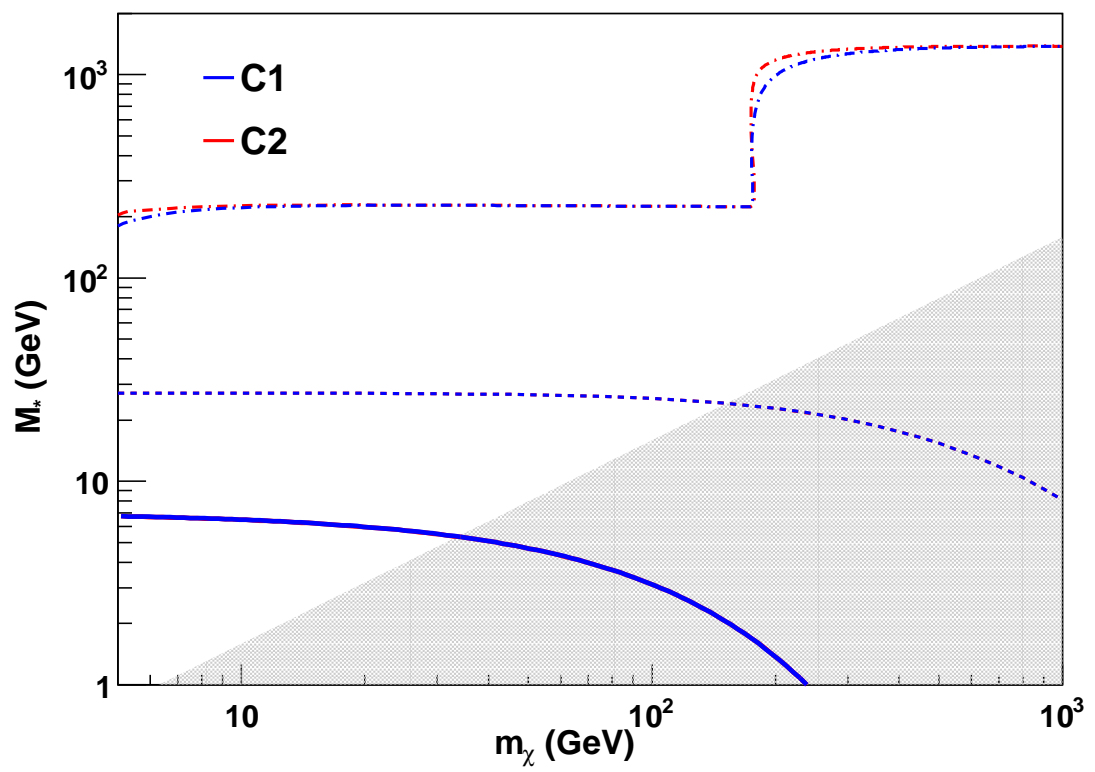

FIG. 12: Same as Fig.8, but for the largely degenerate operators C1 and C2. 


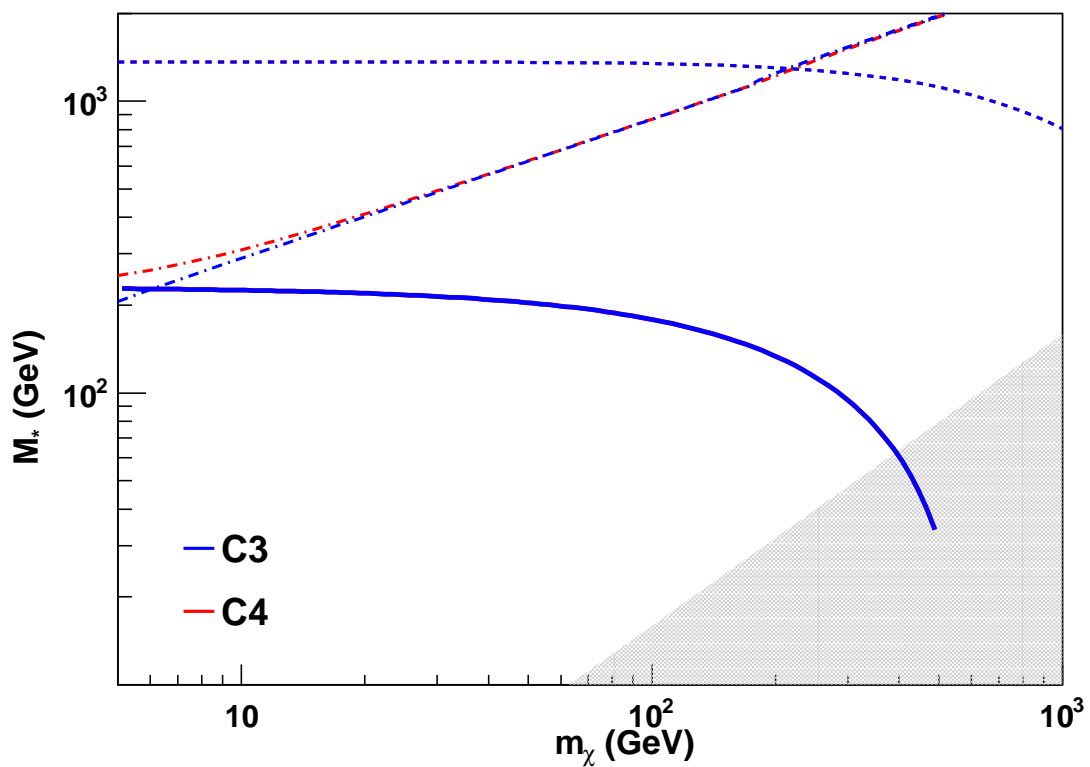

FIG. 13: Same as Fig.8, but for the largely degenerate operators C3 and C4.

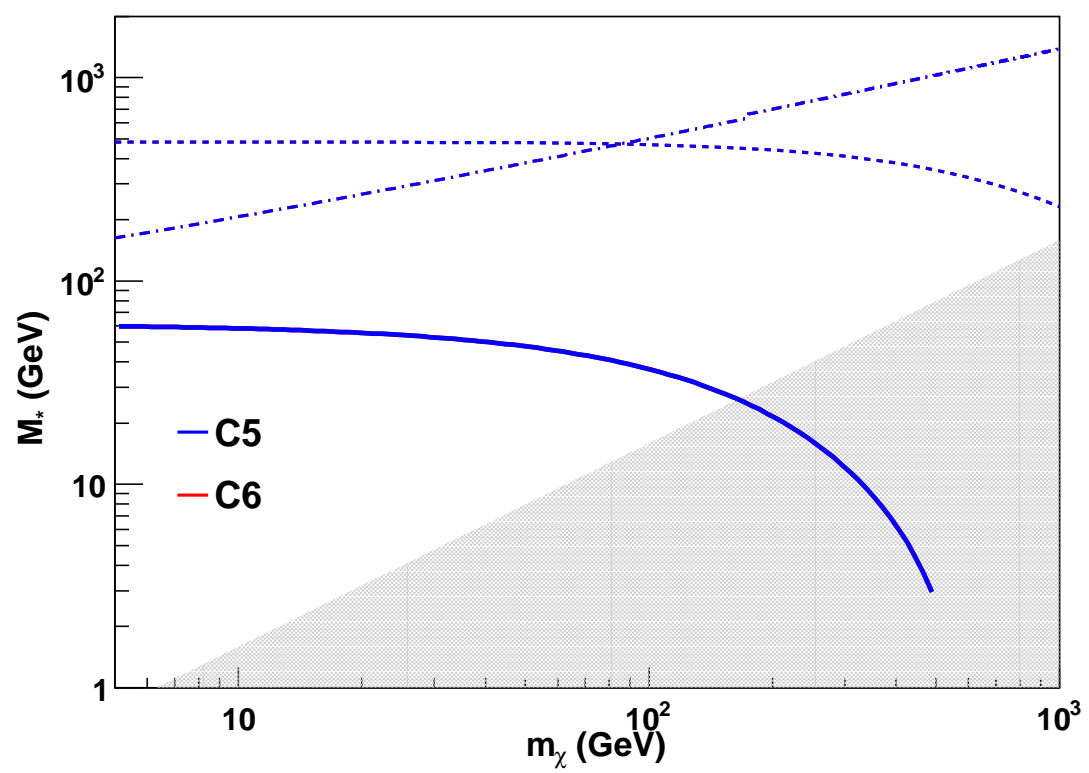

FIG. 14: Same as Fig.8, but for the largely degenerate operators C5 and C6. 


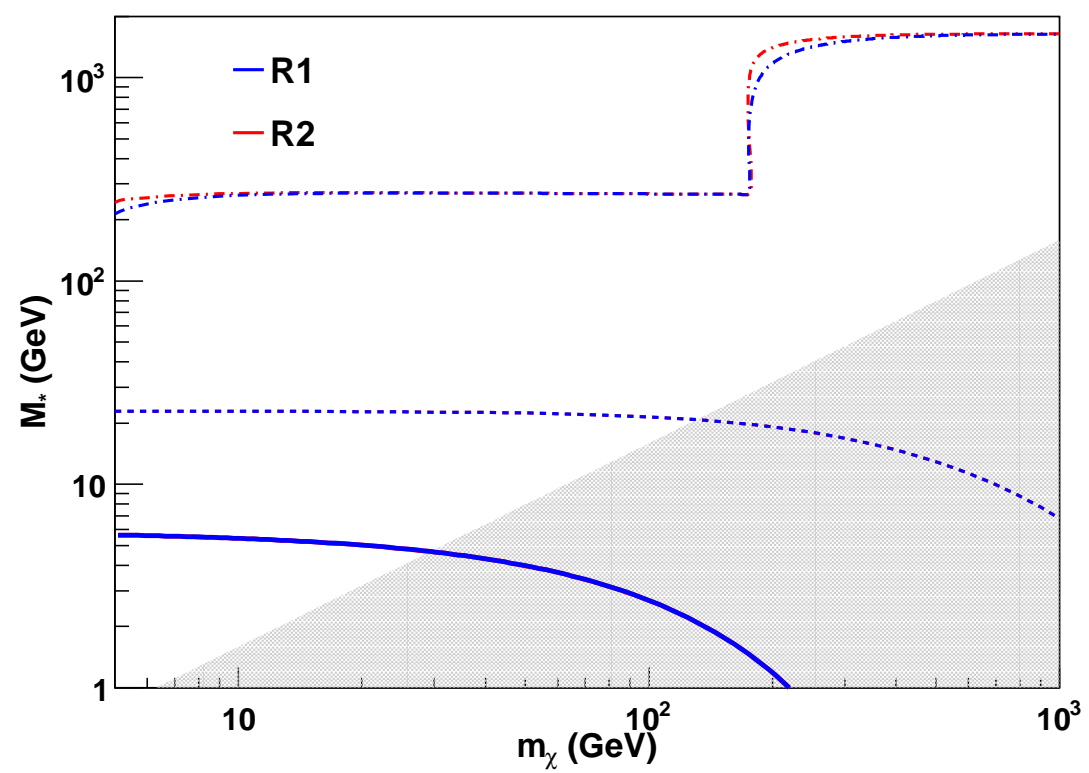

FIG. 15: Same as Fig.8, but for the largely degenerate operators R1 and R2.

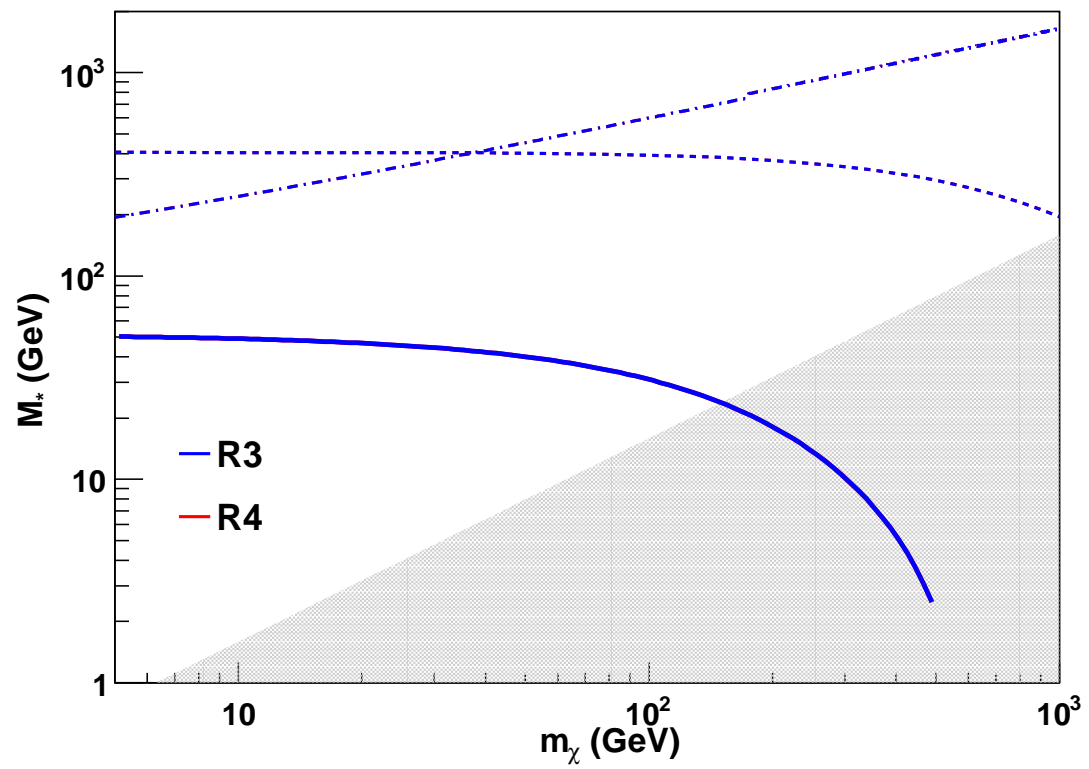

FIG. 16: Same as Fig. 8 , but for the largely degenerate operators R3 and R4. 